Chapter 5

\title{
Kinetics of Amyloid Formation by Different Proteins and Peptides: Polymorphism and Sizes of Folding Nuclei of Fibrils
}

\author{
Oxana V. Galzitskaya, Nikita V. Dovidchenko and \\ Olga M. Selivanova
}

Additional information is available at the end of the chapter

http://dx.doi.org/10.5772/63359

\begin{abstract}
Aggregation of peptides and proteins into amyloid structure is one of the most intensively studied biological phenomena at the moment. To date, there is no developed theory that would allow one to determine what kind of mechanism presents in the given experiment on the basis of aggregation kinetic data. Debates concerning the mechanism of the amyloid fibrils formation and, in particular, the size of the amyloidogenic nucleus are still going on. We created such a theory on the basis of the kinetics of amyloid aggregates formation. In the presented chapter, theoretical and experimental approaches were employed for studding the process of amyloid formation by different proteins and peptides. The current kinetic models described in this chapter adequately describe the key features of amyloid nucleation and growth.
\end{abstract}

Keywords: amyloidogenic regions, amyloid prediction methods, linear and exponential mechanisms of amyloid growth, polymorphism, prion

\section{Introduction}

Many studies show that the generation of an amyloid fibril is a fundamental property of all protein molecules, rather than being limited to a narrow range of the so-called amyloidforming proteins associated with amyloid diseases, as was believed until recently [1, 2]. Normal proteins become toxic upon fibril formation [3]. At the same time, greater cytotoxicity is characteristic of immature water-soluble fibrils compared to mature insoluble amyloid 
fibrils. It seems that the mechanism of toxicity should be similar to that for fibrils formed by different proteins. The structure of toxic immature fibrils is certainly rich in $\beta$-strands and should be universal, because specific antibodies against the precursors of amyloid peptide $A \beta$, associated with Alzheimer's disease, recognize the precursors of amyloid fibrils formed by other proteins with different amino acid sequences [4]. It was demonstrated that samples of blood serum from patients with Parkinson's disease show an autoimmune response to insulin oligomers and fibrils, which should reveal the presence of insulin aggregates in this disease [5]. Fibril formation depends on the experimental conditions and is expedited by denaturants: to aggregate, proteins should be unfolded, at least partly [6]. Preliminary unfolding is not needed for the aggregation of peptides and proteins associated with amyloidosis such as type II diabetes or Alzheimer's and Parkinson's disease, because these proteins are already unfolded under physiological conditions [7]. However, the majority of natively unfolded proteins do not aggregate in vivo [8] suggesting that unfolding is necessary, but not sufficient for the formation of amyloid fibrils. Most likely, there are specific amino acid motifs that are exposed to the solvent and are more prone to aggregation as compared to other regions of the amino acid sequence. Experimental findings support the hypothesis that small protein regions are responsible for the amyloidogenic behavior [9-11].

\section{Identification of aggregation sites in proteins responsible for amyloid formation}

We have done comparison of the prediction results for 30 amyloidogenic proteins [12] using seven methods with experimental data: PASTA2 [13], AmylPred2 [12], Tango [14], MetAmyl [15], Waltz [16], FoldAmyloid [17], ArchCandy [18]. More detail comparison with full list of proteins but without new method Archcandy was presented in our recent paper [19]. One can see that FoldAmyloid (which is a relatively old and simple approach) works better than Waltz and Archcandy. PASTA2 is a new more sophisticated approach that performs best among the non-meta-servers. As evident from Table 1, the accuracy and sensitivity of PASTA2 predic-

\begin{tabular}{|c|c|c|c|c|c|c|c|}
\hline Scoring type & $\begin{array}{l}\text { PASTA2 } \\
90 \% \\
\text { specificity }\end{array}$ & AmylPred2 & Tango & $\begin{array}{l}\text { MetAmyl } \\
\text { high } \\
\text { specificity }\end{array}$ & $\begin{array}{l}\text { Waltz best } \\
\text { perfo- } \\
\text { mance }\end{array}$ & $\begin{array}{l}\text { FoldAmy- } \\
\text { loid }\end{array}$ & $\begin{array}{l}\text { Archcandy } \\
\text { default set- } \\
\text { tings }\end{array}$ \\
\hline Sensitivity & 0.36 & 0.41 & 0.19 & 0.38 & 0.19 & 0.28 & 0.16 \\
\hline Specificity & 0.91 & 0.86 & 0.95 & 0.86 & 0.94 & 0.92 & 0.92 \\
\hline $\begin{array}{l}\text { False regions } \\
\text { predicted as amyloidogen- } \\
\text { ic }\end{array}$ & 38 & 121 & 37 & 88 & 37 & 31 & 15 \\
\hline $\begin{array}{l}\text { No. correctly predicted re- } \\
\text { gions/total }\end{array}$ & $33 / 46$ & $42 / 46$ & $17 / 46$ & $33 / 46$ & $22 / 46$ & $29 / 46$ & $8 / 46$ \\
\hline
\end{tabular}

Table 1. Averaged results of amyloid predictions for 30 proteins by various algorithms. 
tions are only smaller better than those of FoldAmyloid. At the same time, comparison of the overall prediction quality shows only modest improvement, which is due to low sensitivity partly caused by overprediction and partly by the coarseness of the methods.

\section{Possible kinetics schemes for amyloid and prion growth}

To explain the kinetic features of the aggregation process of protein molecules, a large number of schemes were proposed. There are many approaches for describing the kinetics and mechanism of protein aggregation: kinetic, thermodynamic, and other empirical approaches. The purpose of the first two was the best approximation of the experimental data and obtainment of quantitative values of the rate constants describing such processes as nucleation, growth, and all other parts of the aggregation process. At present, there is no consensus whether a protofilament is formed by joining oligomers or by adding monomers. The following questions can be posed: How many different mechanisms exist in the literature for describing the protein aggregation? What is the essence of each mechanism? What mechanisms or models are used in the literature to describe the experimental kinetic curves? What are the similarities between various mechanisms? The large volume of publications describing the formation of amyloid fibrils allows us to reveal two general mechanisms. There are a mechanism of sequential addition of monomers (linear growth) and a mechanism for describing the aggregation of prions (exponentional growth). Most of these approaches somehow go back to the late fifties [20]. In this study, a kinetic scheme for polymerization of actin was proposed. The experimental data indicated that the polymerization process is very similar to the condensation reaction: The reaction occurs only when the monomer concentration exceeds the critical threshold. The assumption was justified. The cooperative polymerization reaction was confirmed by two facts: (1) The reaction rate in the early stages of the reaction increased upon increasing the concentration of actin; (2) an addition of seed with an already formed unit led to an immediate transition of all free actin to an aggregate. In 1974, Hofrichter et al. [21] used the mechanism of sequential monomer addition to the aggregate to explain the formation of hemoglobin fibrils in sickle cell anemia. To explain the observed effect of "extreme autocatalysis" and strong concentration dependence, Ferrone with the co-authors developed a model of "heterogeneous nucleation" [22]. This model assumes the following sequence of events: First, in the normal course of nucleation, fibrils, and then, subsequent fibrils may form on its surface. This model is included as an equation for the first stage-the homogeneous nucleation, and for the second step-heterogeneous nucleation; the equations were solved numerically. The notion of heterogeneous nucleation was a new and important contribution to the development of the theory of protein aggregation. In 1983, Frieden and Goddette [23] used a model of sequential monomer addition to the unit to describe the process of protein polymerization, with the proviso that each step of monomer addition had its own reaction rate constant. In 1986, Goldstein and Stryer [24] modified the model of sequential addition of monomers to the unit to describe the process of protein polymerization. They identified the nucleus as a "seed" of size s, after the formation of which the kinetic constants are changed. Using protein amylin, it was shown that nucleation can occur in two ways: fibril- 
independent (or primary nucleation) and fibril-dependent (secondary nucleation) paths. The contribution of each of these processes depends on the external interfaces (or surfaces). In the presence of such a surface, the primary nucleation mechanism is dominating; in the absence of such a surface, the secondary nucleation mechanism is dominating [25]. Heterogeneous nucleation can be seen from another point of view, as an autocatalytic growth in mature seeds. It was not shown experimentally whether seeds are formed on the existing surface of the unit, or the growth simply continues from the surface of the same aggregate. In 1967, Griffith [26] suggested three possible theoretical mechanisms that could explain the self-replication agent causing "scrapie" sheep (scrapie). In 1982, Prusiner [27] was able to provide enough experimental evidence that the "scrapie" infectious agent is the protein called a prion. In 1991, Prusiner suggested the scheme of transfer and development of prion infection [28]. Based on the Prusiner's work, in 1993, Lansbury his work published [29], in which the mechanism of sequential monomer addition to the seed was applied for analysis of kinetic data.

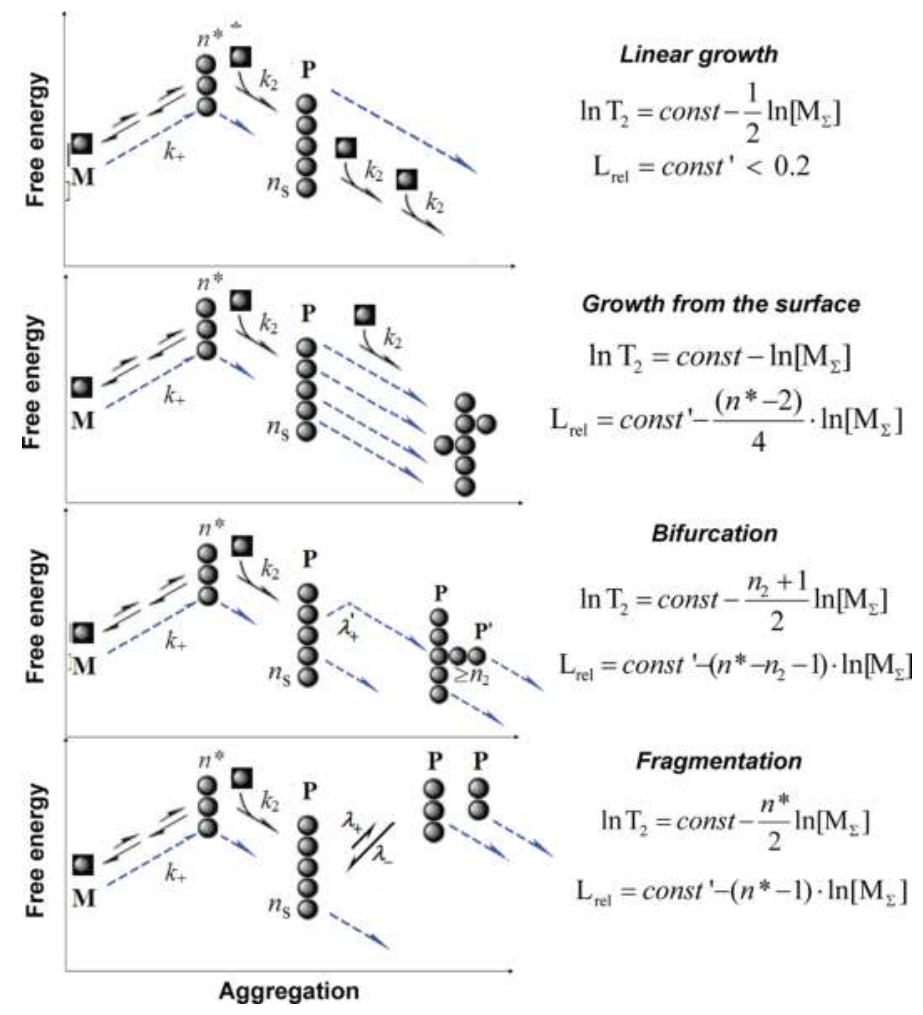

Figure 1. Alternative scenarios for amyloid growth and corresponding kinetic parameters. $\mathrm{T}_{2}$ is the time of inclusion of all monomers into the aggregate, $\mathrm{L}_{\mathrm{rel}}$ is the ratio between the duration of the lag phase and the time of inclusion of all monomers into the growing polymer, $\left[\mathrm{M}_{\Sigma}\right]$ is the total monomer concentration, $\mathrm{n}^{*}$ is the size of the primary nucleus, and $\mathrm{n}_{2}$ is the size of the secondary nucleus. $\mathrm{n}_{\mathrm{S}}$ is the smallest number of monomers in the stable polymer. Modified from [33]. 
In 2004 in the paper by Collins et al. [30], the formation kinetics of sup35p prion was studied. It was shown that amyloid aggregate growth occurs upon monomer addition to the fiber ends. Also the authors performed experiments with agitation and observed catalysis due to fiber fragmentation, so their kinetic model consisted of three steps: at the first step, oligomers are formed; at the second step, amyloidal seeds are formed through nucleation-conversion, after that the autocatalytic growth occurs where autocatalysis takes place because of fragmentation. Thus, major schemes of amyloid aggregates formation were drawn. In general, schemes can be summed up into the following reaction: out of a pool of monomer nuclei (the most unstable species on the reaction path with the largest free energy) are formed, which then are somehow converted into seed (the smallest stable amyloid aggregate) which is able to attach monomers in a way that monomer free energy only decreases, the result of which is that after some time, all the monomers become associated into fibrils. In some cases, catalysis of reaction occurs through secondary nucleation or fragmentation. In the presence of a catalyst, the amyloidal growth regime can be named "exponential," whereas in the absence of catalysis, the amyloidal growth can be named linear. It should be mentioned that in this general scheme, it is not important what one means by the words "monomer protein" - the monomer itself or some protein aggregate. Despite overall success in the understanding of possible mechanisms hidden beneath amyloidal aggregation, the relation of the kinetic models to the experimentally measurable values was still unclear. Thus, the authors of the paper [31] have shown that despite possible different mechanisms of aggregation, all of the kinetic experimental data could be successfully approximated by a simple sigmoid curve. Fortunately in the same year, the careful introspection of the behavior of the logarithm of lag time versus logarithm of concentration for several amyloidogenic agents by Knowles et al. [32] revealed that different amyloidogenic agents possess a similar linear dependence in those coordinates and such dependence is $\mathrm{T}_{\text {lag }} / \mathrm{T}_{\operatorname{lag}_{0}} \approx\left(\mathrm{C} / \mathrm{C}_{0}\right)^{-1 / 2}$, where $\mathrm{T}_{\operatorname{lag}_{0}}$ and $\mathrm{C}_{0}$ are values corresponding to the smallest concentration. In the paper Dovidchenko et al. [33], the authors studied the variance in the mechanisms within scheme monomer ->nucleus->fibril growth with/without catalysis. In the work, the authors were lucky to find bridges between values which can be calculated directly from experimental data-characteristic times such as lag-time $\mathrm{T}_{\text {lag }}$ time of inclusion of all monomers into aggregate $\mathrm{T}_{2}$ and relation $\mathrm{L}_{\mathrm{rel}}=\mathrm{T}_{\mathrm{lag}} / \mathrm{T}_{2}$ and mechanism of amyloidogenesis. It turned out that through the study of the concentration dependence of amyloid formation, one can estimate nuclei sizes (the size of the primary nucleus and size of the secondary nucleus if secondary nucleation takes place) and possible mechanisms. For example, linear growth (when no catalysis occurs) has a very narrow range of conditions: $\mathrm{L}_{\mathrm{rel}}$ should be independent of the logarithm of concentration and its value should not exceed 0.2. In the case of exponential mechanism, one should plot a graph of two dependencies: $\ln \mathrm{T}_{2}$ versus $\ln \left[\mathrm{M}_{\Sigma}\right]$ and $\mathrm{L}_{\text {rel }}$ versus $\ln \left[\mathrm{M}_{\Sigma}\right]$, where $\mathrm{M}_{\Sigma}$ is the initial concentration of monomers. As one can see from Figure 1, the dependencies should be linear in those coordinates and thus the calculation of the tangent coefficient of a line drawn through experimental points will point out what mechanism takes place in the given reaction. However, several tangent coefficients (for example, $-1 / 2$ which can be either the result of a fragmentation mechanism or a bifurcation mechanism without secondary nucleation) are special cases and can be obtained in 
different scenarios; for those cases, additional experiments are needed to exclude excess mechanism variants.

\section{Polymorphism of amyloid fibrils}

The fully matured fibers are not the most toxic species. The importance of intermediate species that define the pathway, toxicity, and even the type of fibers formed at the end should be emphasized. Usually electron microscopy (EM) is used for comprehensive characterization of the amyloid formation and for control of the amyloid morphology. The probes are collected for analyzing periodically over duration of all experiments. The main results of EM experiments at each of the time intervals are the properties of time-dependent structures and their variability (polymorphism). These analyses usually are performed using both commercial and recombinant samples. The use of EM in studying the process of amyloid formation is due to the importance of polymorphism for determining the protein folding pathways. On the other hand, protein folding pathway depends strongly on its amino acid sequence. Further, the resulting protein conformation affects the formation of amyloid fibrils, and the same polypeptide sequence may correspond to the fibrils of different morphologies [34]. The fibril polymorphism is potentially important for the human disease progression, as it may be the reason for natural variability of amyloid-related pathologies such as amyloidosis of light chains of immunoglobulins (AL) and the phenomenon of strain variability of prion infection $[34,35]$. The EM images of amyloid fibrils show insignificant morphological differences, and the fibrils are often represented as twisted or parallel clusters of thin protein filaments. One should note also that polymorphism is a serious obstacle in studying fibril structures. A mixture of fibrils of different morphology is always present in solution, and several different types of fibrils can be seen under the same conditions. This creates a problem for image analyses because it requires separation of fibrils by their morphology and performing separate analyses of each type of insulin, $A \beta$ peptide, $\beta_{2}$-microglobulin, glucagon, amylin, calcitonin, etc. It is not completely excluded that different fibril morphologies are due to similar but not identical fibril formation pathways. The differences may be formed at the initial steps of fibril formation including the step of nucleation. The size of the nucleus of a protofibril is still unknown for almost all proteins and peptides. Nucleation is the limiting step of the amyloid fibril formation which can start only when the concentration of amyloid proteins exceeds the critical one. After the nucleation, the fibrils grow rapidly. Proteins may have different sizes of the protofibril nuclei (see the next sections). Also, the conditions at which the fibrils are formed may affect its size. This may result in the formation of the fibrils of different morphologies. In other words, we assume that the fibril formation depends on the nucleation step and goes through different pathways, thus determining the final fibril morphology. Note also that both the morphology and molecular structure are sensitive to small differences under conditions when the fibrils are growing in de novo preparations (under fixed $\mathrm{pH}$, temperature, buffer composition, and peptide concentration). 


\subsection{Polymorphism of $A \beta$ fibrils}

It was shown previously that peptide $\mathrm{A} \beta 40$ forms fibrils of different morphologies even under the same conditions. For the fibrils formed without mixing, the dominating morphologies are twisted fibrils, while the activated fibrils will be less twisted yet inclined to form lateral associations. The inactivated twisted fibrils are the most toxic for the cells [36]. Therefore, it is important to study all possible formations including oligomers and fibrils, which determine the natural polymorphism. Moreover, it was shown previously that the fibrils associated with diseases have the same nucleation center structure called a $\beta$-arcade. The $\beta$-strands in these
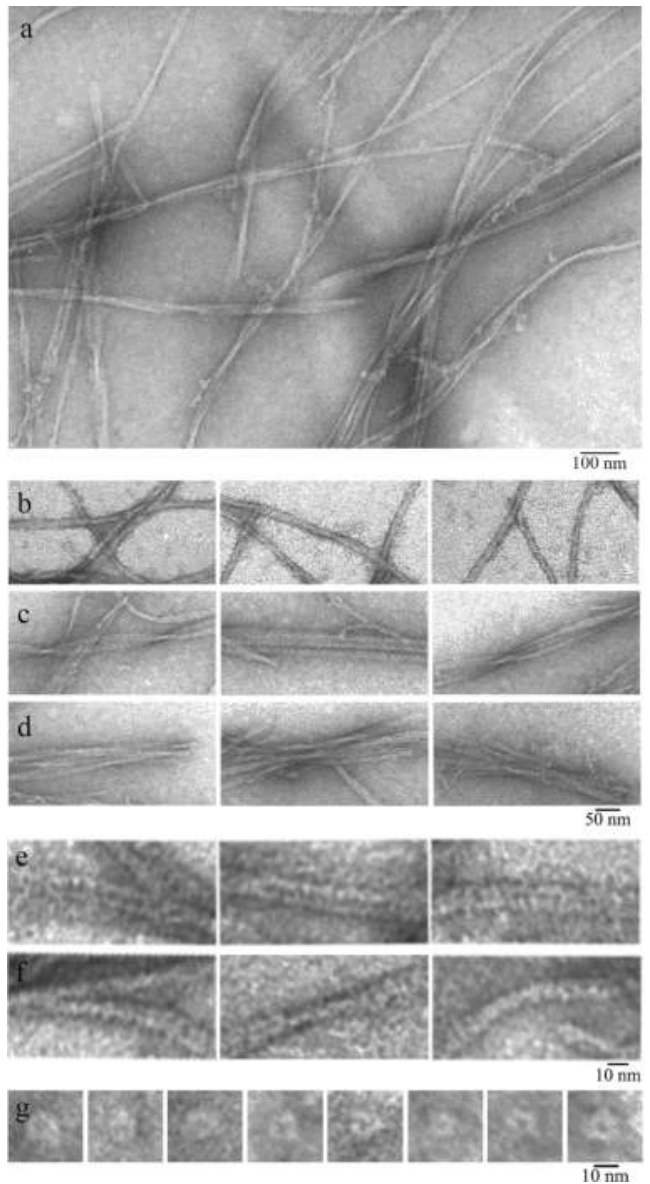

Figure 2. Electron microscopy images of recombinant $\mathrm{A} \beta 40$ peptide $\left[0.2 \mathrm{mg} / \mathrm{mL}, 27 \mathrm{~h}\right.$ incubation at $25^{\circ} \mathrm{C}, 50 \mathrm{mM}$ Tris$\mathrm{HCl}(\mathrm{pH} 7,5)]$. (a) General view (field) of recombinant A $\beta 40$ peptide after $27 \mathrm{~h}$ of incubation. (b-d) Gallery of types of A $\beta 40$ peptide fibril images at higher magnification: (b) single fibrils; (c) ribbon-like type of fibrils; (d) bundle-like type of fibrils; (e, f) single fibrils: (e) at horizontal packing; (f) bending sites of the single fibrils. (g) Gallery of ring oligomers. 
structures are parallel to each other. It is interesting to note that this kind of a nucleus structure is not found in normal proteins and the $\beta$-strands are antiparallel to each other [37].

\subsubsection{Morphology of recombinant Aß40 peptides}

Figure 2 shows the preparation of recombinant $\mathrm{A} \beta 40$ peptide after $27 \mathrm{~h}$ incubation at $25^{\circ} \mathrm{C}$ at $50 \mathrm{mM}$ Tris $-\mathrm{HCl}(\mathrm{pH} 7,5)$ and $\mathrm{C}=0.2 \mathrm{mg} / \mathrm{ml}$. Polymorphism of generated fibrils is seen. In addition to single fibrils of several microns in length with a diameter of about 8-9 $\mathrm{nm}$, there are also fibrils, forming bands of different widths, and bundles. It seems that single fibrils consist of two filaments. However, under high magnification, it is seen that the fibrils are formed of rounded ring structures. The matter is that upon staining the preparations, the staining agent dyes not only their contours, but also flows into different cavities and openings. This should be taken into account when interpreting EM images; otherwise, a tobacco mosaic virus fibril would consist of two parallel packed short filaments of $300 \mathrm{~nm}$ long. In the case of the $\mathrm{A} \beta 40$ peptide preparation, under high magnification, it is also seen that the staining agent flows into the openings of ring structures. These ring oligomer structures have a diameter of about 8-9 $\mathrm{nm}$ (the width of a single fibril) and a diameter of the internal opening of about $3 \mathrm{~nm}$. Ring structures or A 340 oligomers form single fibrils in such a way, that ring oligomers are layered on each other. If a fibril is absorbed on the formvar film sideways, we see a thinner fibril with a diameter of about $4 \mathrm{~nm}$. This size demonstrates an approximate height of a ring-structured oligomer. As a matter of fact, the height is smaller, because the degree of
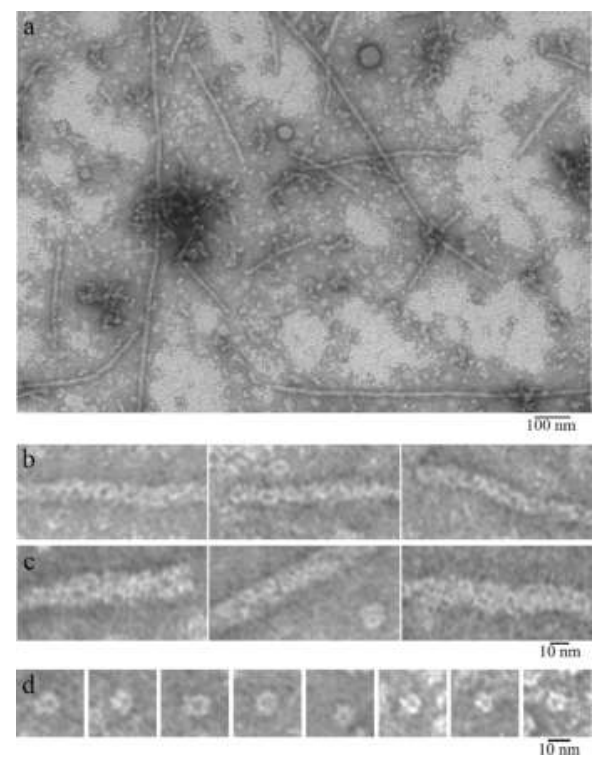

Figure 3. Electron microscopy images of A $\beta 42$ peptide from Invitrogen (lot \#73269340A). The sample was incubated at $37^{\circ} \mathrm{C}$ (water). (a) General view of the sample at $97-\mathrm{h}$ incubation. (b, c) Gallery of types of A $\beta 42$ peptide fibrils at higher magnification: (b) single thin and (c) thick fibrils. (d) Gallery of ring oligomers. 
overlapping of ring-structured oligomers should be taken into account. The greater is the overlapping, the larger is the height of such packing of fibrils.

\subsubsection{Morphology of synthetic A 342 peptide (Invitrogen, lot \#73269340A)}

The EM image of A $\beta 42$ peptide (Invitrogen, lot \#73269340A) (Figure 3) shows that after $97 \mathrm{~h}$ of incubation at $37^{\circ} \mathrm{C}$ (water), fibrils of several microns long are formed. Polymorphism of fibrils is observed. Thin fibrils with a diameter of about $7-8 \mathrm{~nm}$ and more are seen, and more pronounced polymorphism of the generated fibrils is observed (Figure 3). At the same time, fibrils of different diameters are present: thin ones of about 7-8 nm and wider fibrils up to $15 \mathrm{~nm}$. Moreover, rounded ring-structured oligomers of about $8 \mathrm{~nm}$ in diameter can be seen; the internal diameter of the ring (the diameter of the hole) is about $3 \mathrm{~nm}$ (Figure $3 \mathrm{~d}$ ). At magnification, it can be seen that an oligomer consists of 4-6 smaller rounded particles (Figure 3d). It is notable that both thin and thick fibrils are formed of such ring-structured oligomers. Thin fibrils are characterized by more ordered interaction of separate oligomers, while thick fibrils are generated due to random sticking of additional oligomers along the fibril axis. Because of this way of generation, fibrils look uneven in EM images with a tendency to branching [38].

Thus, using the EM method, we have demonstrated that fibrils of these peptides are formed by association of rounded ring structures. Negatively stained TEM images of fibrils extracted from AD brain tissues [39] are very similar to our EM results. Moreover, similar structures are observed elsewhere [40].

\subsection{Polymorphism of insulin fibrils}

Insulin has a high tendency to aggregation and forms fibrils. Insulin fibrils were first described in 1940 by Waugh [41]. At present, the conditions leading to the formation of active insulin fibrils are known [42]. Insulin fibrillation occurs upon various environmental changes $(\mathrm{pH}$, ionic conditions, temperature, organic solvents, and agitation); it also depends on the methods for producing insulin, its delivery, and storage. It should be noted that the human insulin fibrillation is slower than that of the best studied bovine insulin [42-47].

Currently, all insulin preparations for therapeutic purposes are prepared in the form of a Zncontaining hexamer, since in this condition it is more stable and resistant to the formation of fibrils. When treating diabetes, insulin penetrates into muscles by injection, where it typically forms deposits. The large size of the insulin hexamer slows its adsorption, because the hexamer must go through the dimers in the monomeric state and only in this way penetrate into the bloodstream. This leads to slowing down the interaction with insulin receptors and the need to take insulin $1-1.5 \mathrm{~h}$ before mealtime.

Despite intensive studies of fibrillogenesis of insulin, there is no generally accepted scheme for the formation of mature fibrils. The main difficulties arise in interpreting the beginning of the fibrillation process. Polymerization of insulin begins from the transition of the monomer protein from the native state to the partially unfolded conformation which can be amyloidogenic. The strong dependence of the reaction of polymerization on various external parame- 
ters leads to the formation of insulin fibrils with different morphology, which complicates the analysis of the general scheme of fibril formation.

For electron microscopy (EM) investigations, insulin samples were used at a concentration of $0.2 \mathrm{mg} / \mathrm{ml}$. Zinc-free recombinant human insulin was obtained from "Gerofarm-Bio" (Obolensk, Russia) and was checked by the tandem mass spectrometry technique (LCQ Deca $\mathrm{XP}$, Thermo Finnigan, USA). According to the EM data on polymerization of human insulin, the mature protein fibrils appeared only after $8 \mathrm{~h}$ of insulin incubation. Active polymerization of insulin occurs up to $10-11 \mathrm{~h}$ of incubation. This is accompanied by the elongation of the fibrils for both samples up to $10-12 \mu \mathrm{m}$. The fibrils interact laterally with each other, resulting in the formation of big clusters composed from ribbons, bundles, and twisting bundles (Figure 4). Protein aggregation is the result of increasing the incubation time up to $24 \mathrm{~h}$. According to the EM data, the number of large fibril clusters increases in the samples after $24 \mathrm{~h}$, but the length of the fibrils does not change (remains about 10-12 $\mu \mathrm{m}$ ) [48].

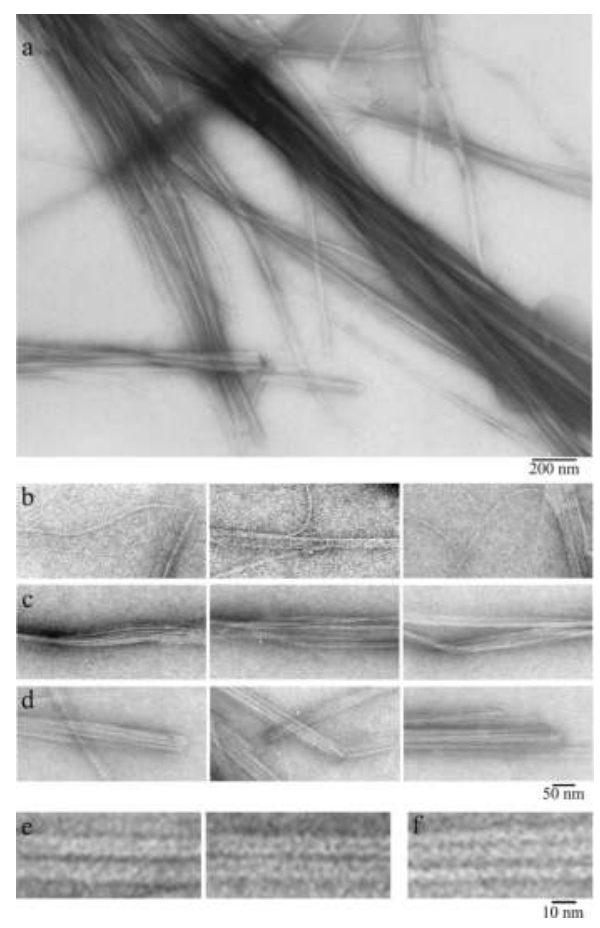

Figure 4. Electron microscopy images of human zinc-free recombinant insulin $\left(0.2 \mathrm{mg} / \mathrm{ml}, 24 \mathrm{~h}\right.$ incubation at $37^{\circ} \mathrm{C}, 20 \%$ acetic acid ( $\mathrm{pH}$ 2.0), $140 \mathrm{mM} \mathrm{NaCl}$ ). (a) General view (field) of human insulin after $24 \mathrm{~h}$ of incubation. (b-d) Gallery of images of types of insulin fibrils at higher magnification: (b) single fibrils; (c) ribbon-like type of fibrils; (d) bundle-type of fibrils. (e, f) The thinnest fibrils: (e) at horizontal packing; (f) at vertical packing of ring oligomers. 


\section{Example of amyloid growth by the linear nucleation-elongation mechanism (actin, apolipoprotein C-II)}

Most quantitative models for linear polymerization stem from the work performed more than half a century ago [20] proposing a kinetic model for actin polymerization. Only two of the proteins (apolipoprotein C-II [49]: $\mathrm{L}_{\text {rel }}<0.1$ with the primary nucleus size $n^{*} \approx 4-5$ and actin with $n^{*} \approx 3$ ), studied in our analysis, exhibit linear growth. Formation of actin filaments occurs by linear growth - fibrils increase only due to attachment of monomers to the aggregate ends. This is supported by literature data [50] and by analysis of kinetic data (Figure 1). According to the theory advanced in [33] for linear growth (observed in actin), the characteristic time of the relative lag-period $L_{\text {rel }}$ does not exceed 0.2 and is independent of $\ln \left[\mathrm{M}_{\Sigma}\right]$ as in the case of actin (Figure 5) [50]. The size of the primary nucleus was calculated from the slope to be $n=3.46 \pm 0.28$.
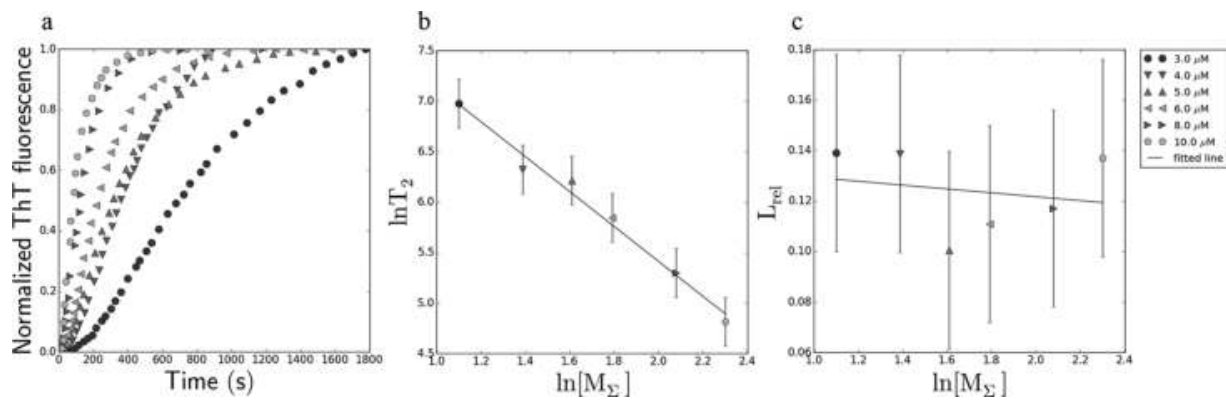

Figure 5. (a) Dependence of normalized ThT fluorescence on time for actin [50]. (b) Dependence of $\mathrm{L}_{\text {rel }}$ on $\ln \mathrm{T}_{2}$. (c) Dependence of $L_{\text {rel }}$ on $\ln \left[\mathrm{M}_{\Sigma}\right]$. The slope angle of the fitted line is about -0.01 , that is, $\mathrm{L}_{\mathrm{rel}}$ is independent of $\ln \left[\mathrm{M}_{\Sigma}\right]$. All $\mathrm{L}_{\text {rel }}$ values are also $<0.2$ which, according to the models of formation of fibrillar aggregates, means that the formation of actin fibrils proceeds by the linear mechanism (see [33]). The errors are computed with the Student's coefficients corresponding to 0.95 confidence level.

\section{Examples of amyloid growth by the exponential mechanism and sizes of folding nuclei of fibrils formed by different agents}

In order to estimate the size of fibril nucleus (the most unstable state on the monomer to the fibril pathway) and a possible scenario for the formation of aggregates, it is necessary to make a number of kinetic experiments, where the only variable parameter is the monomer concentration. Characteristic times $\mathrm{T}_{\mathrm{lag}}$ (the lag-period duration), $\mathrm{T}_{2}$ (the time of transition of all monomers into an aggregate), and $\mathrm{L}_{\text {rel }}$ (the $\mathrm{T}_{\text {lag }} / \mathrm{T}_{2}$ ratio) are calculated for each experimental curve. It was demonstrated that the dependences of $\ln \mathrm{T}_{2}$ and $\mathrm{L}_{\text {rel }}$ on $\ln \left[\mathrm{M}_{\Sigma}\right]$ (the logarithm of the initial concentration of monomers) are linear, with gradients that can be used for the computation of fibril nucleus sizes (including those of non-amyloid type) and for elucidation of the mechanism of aggregate formation. 
If $\mathrm{L}_{\mathrm{rel}}>0.2$, the linear growth model is excluded, and, independently of $\ln \mathrm{T}_{2}$ and $\mathrm{L}_{\text {rel }}$ dependence on $\ln \left[\mathrm{M}_{\Sigma}\right]$, only the exponential growth scenario remains. The dependences of $\ln \mathrm{T}_{2}$ on $\ln \left[\mathrm{M}_{\Sigma}\right]$ and $\mathrm{L}_{\mathrm{rel}}$ on $\ln \left[\mathrm{M}_{\Sigma}\right]$ are formally different for different exponential scenarios (i.e., "growth from the surface," "fragmentation," and "bifurcation"). However, it is impossible, for example, to distinguish the bifurcation from the fragmentation scenario from the kinetics alone if the size $n_{2}$ of the secondary nucleus of bifurcation is zero; this implies the need for direct observations of fibril shapes to distinguish these cases (see Refs. [33, 48] for more details).

\subsection{Insulin}

Now we can present three general schemes of insulin amyloid formation, which differ by the size of folding nuclei of fibrils. For the first type, the size of nucleus is one monomer [44, 5153]. For the second scheme of fibril formation, the size of nucleus is two monomers and the dimers are precursors of fibrils $[5,54,55]$. For the third scheme of polymerization, oligomers are precursors of fibrils, and the size of oligomer can vary from three to six monomers $[42,43$, 56-58].

The basis for choosing between different mechanisms of formation of fibrils is to detect various soluble oligomers. However, at low concentrations and at early times, it is difficult to accomplish accurate detection of the initial aggregates. For this purpose, a variety of optical methods, as well as Cryo-transmission-EM, atom force microscopy, and small-angle X-ray scattering are used [55,57-59]. However, all these methods are not quantitative, have no reliable standards, do not provide the full size distribution, may yield abnormal results (with microscopic approaches-interaction with the surface during adsorption of samples), and require the analysis of the results of certain programs to prove the statistical significance [52]. Although all these methods provide valuable information, none of them can convincingly demonstrate how an unstable nucleus grows to a stable seed for further growth of protofibrils, and many experimental and theoretical models are based on this assumption.
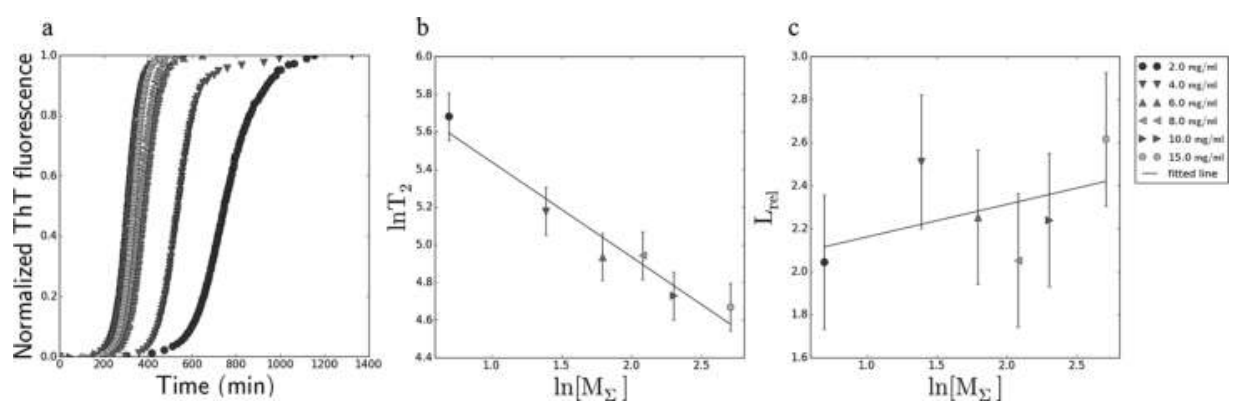

Figure 6. (a) Dependence of normalized ThT fluorescence on time for insulin [48]. The approximation line calculated for insulin amyloid formation with the experimental data from (a) by the least-squares method plotted in coordinates (b): $\left\{\ln \mathrm{T}_{2}, \ln \left[\mathrm{M}_{\Sigma}\right]\right\}$ and (c): $\left\{\mathrm{L}_{\mathrm{rel}}, \ln \left[\mathrm{M}_{\Sigma}\right]\right\} ;\left[\mathrm{M}_{\Sigma}\right]$ is in $\mathrm{mg} / \mathrm{ml}, \mathrm{T}_{2}$ in min. The tangent coefficients of the approximation lines are $-0.51 \pm 0.13$ for $d\left(\ln T_{2}\right) / d\left(\ln \left[\mathrm{M}_{\Sigma}\right]\right)$ and $0.15 \pm 0.31$ for $\mathrm{d}\left(\mathrm{L}_{\mathrm{rel}}\right) / \mathrm{d}\left(\ln \left[\mathrm{M}_{\Sigma}\right]\right)$; the errors are computed with the Student's coefficients corresponding to 0.95 confidence level. 
The growth of amyloid fibrils was studied by thioflavin T (ThT) fluorescence, which drastically increases when ThT is bound to fibrils (Figure 6a). The renormalized curves, presented in Figure 6a, are very similar to one another. They have large lag periods, thus excluding the linear growth scenario. The obtained analytical solution and computer modeling allowed us to determine the size of the nucleus from the experimentally obtained concentration dependences of the relationship between the lag-time duration and the time of growth of amyloid fibrils [33]. Figure $6 \mathrm{~b}, \mathrm{c}$ illustrates determination of the fibrillation scenario and the nuclei sizes $\left(n^{*}, n_{2}\right)$ for human insulin (see Table 2$)$ [48]. The value $d\left(\ln _{2}\right) / d\left(\ln \left[\mathrm{M}_{\Sigma}\right]\right) \approx-0.5$ is inconsistent with the "growth from the surface" model but consistent with "linear growth" (which, though, should have $\mathrm{L}_{\text {rel }}<0.2$, which is inconsistent with $\mathrm{L}_{\text {rel }}$ ), "fragmentation" (which, though, is inconsistent with EM images shown in Figure 4), and "bifurcation" (consistent with the EM images), with the secondary nucleus size $n_{2}=0$ calculated as $-1-d\left(\ln \mathrm{T}_{2}\right) / d\left(\ln \left[\mathrm{M}_{\Sigma}\right]\right)$; the primary nucleus size $n^{*}=1+n_{2}-d\left(\mathrm{~L}_{\mathrm{rel}}\right) / d\left(\ln \left[\mathrm{M}_{\Sigma}\right]\right)=1$ (see Table 2 ). According to the elaborated theory, it means that the size of the primary nucleus corresponds to one monomer.

\begin{tabular}{|c|c|c|c|c|c|c|}
\hline $\begin{array}{l}\text { Amyloid-forming } \\
\text { protein or peptide and a } \\
\text { ref. to its experimental } \\
\text { study }\end{array}$ & $\begin{array}{l}{\left[\mathrm{M}_{\mathrm{\Sigma}}\right],} \\
\mathrm{mg} / \mathrm{ml} \\
(\mathrm{min}-\max )\end{array}$ & $\begin{array}{l}\ln \left(T_{2} / \min \right) \text { at } \\
\text { various }\left[M_{\Sigma}\right] \\
(\min -\max )\end{array}$ & $\begin{array}{l}\mathrm{L}_{\mathrm{rel}} \text { at } \\
\text { various } \\
{\left[\mathrm{M}_{\Sigma}\right]} \\
\text { (min-max) }\end{array}$ & $\begin{array}{l}n^{*} \pm \Delta n^{*} \\
\text { primary } \\
\text { nucleus }\end{array}$ & $\begin{array}{l}n_{2} \pm \Delta n_{2 \prime} \\
\text { secondary } \\
\text { nucleus }\end{array}$ & $\begin{array}{l}\text { Model that follows } \\
\text { from the } L_{\text {rel }} \text { and } \ln T_{2} \\
\text { on }\left[M_{\Sigma}\right] \\
\text { dependencies }\end{array}$ \\
\hline $\begin{array}{l}\text { Human insulin [48] } \\
\left(37^{\circ} \mathrm{C}\right)\end{array}$ & $2.0-15$ & $4.71-5.70$ & $1.78-2.48$ & $0.86 \pm 0.44$ & $0.01 \pm 0.13$ & Exponential \\
\hline $\begin{array}{l}\text { Human insulin [65] } \\
\left(45^{\circ} \mathrm{C}\right)\end{array}$ & $2.5-20$ & $3.60-4.18$ & $5.17-5.56$ & $1.13 \pm 0.19$ & $-0.48 \pm 0.16$ & Exponential \\
\hline $\begin{array}{l}\text { LysPro human insulin } \\
{[48]\left(37^{\circ} \mathrm{C}\right)}\end{array}$ & $2.0-15$ & $5.78-4.81$ & $2.04-4.38$ & $0.63 \pm 1.04$ & $-0.29 \pm 0.24$ & Exponential \\
\hline
\end{tabular}
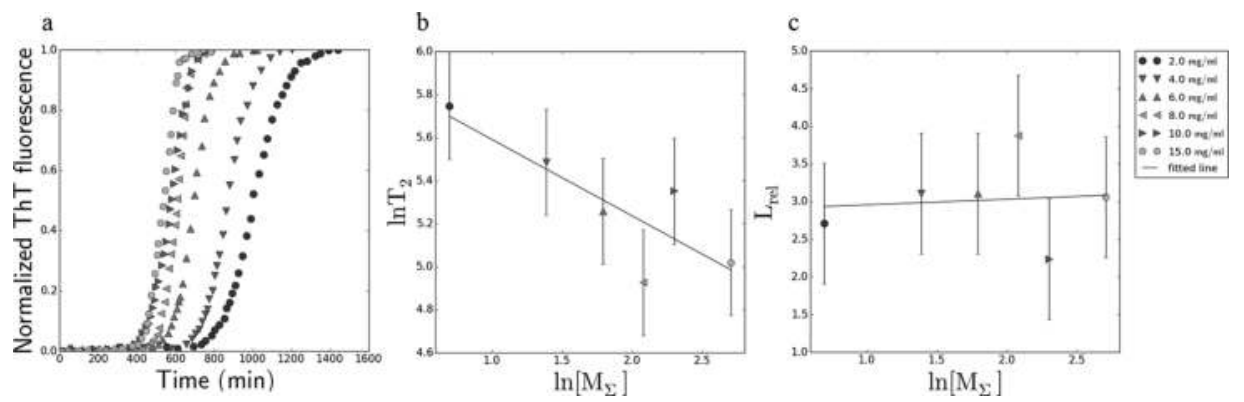

Figure 7. (a) Dependence of normalized ThT fluorescence on time for LysPro insulin [48] The approximation line calculated for LysPro insulin amyloid formation with experimental data from (a) by the least-squares method plotted in coordinates (b): $\left\{\operatorname{lnT}_{2}, \ln \left[\mathrm{M}_{\Sigma}\right]\right\}$ and $(\mathrm{c}):\left\{\mathrm{L}_{\mathrm{rel}}, \ln \left[\mathrm{M}_{\Sigma}\right]\right\} ;\left[\mathrm{M}_{\Sigma}\right]$ is in $\mathrm{mg} / \mathrm{ml}, \mathrm{T}_{2}$ in $\min$. The tangent coefficients of the approximation lines are $-0.35 \pm 0.25$ for $\mathrm{d}\left(\ln _{2}\right) / \mathrm{d}\left(\ln \left[\mathrm{M}_{\Sigma}\right]\right)$ and $0.07 \pm 0.80$ for $\mathrm{d}\left(\mathrm{L}_{\mathrm{rel}}\right) / \mathrm{d}\left(\ln \left[\mathrm{M}_{\Sigma}\right]\right)$; the errors are computed with the Student's coefficients corresponding to 0.95 confidence level. 
Table 2. The range of $\mathrm{L}_{\text {rel }}$ and $\ln \mathrm{T}_{2}$ values, the sizes of the primary $\left(n^{*}\right)$ and secondary $\left(n_{2}\right)$ nuclei and the amyloid growth scenarios obtained from experimental kinetic data alone.

\subsection{LysPro human analog insulin}

We have investigated the kinetics of formation of fibrils by monomeric rapid-acting insulin LysPro [48]. According to our data, fibril formation of the insulin analog at $\mathrm{pH} 2$ proceeds by $5 \mathrm{~h}$ longer than that of the recombinant human insulin. These data seem to us important because the rate of aggregation of insulin preparations is an important health problem not only in the production and storage of insulin, but also for its frequent injections. In this regard, the process of fibrillation should be analyzed for all developed analogs, already prepared for clinical trials and for mass production. Examination of EM images of growing insulin LysPro fibrils shows that they occurred with a kind of the "bifurcation plus lateral growth" scenario [48]. The growth of amyloid fibrils was studied (Figure 7) by thioflavin T (ThT) fluorescence, which drastically increases when ThT is bound to fibrils. The dependence of $\mathrm{L}_{\text {rel }}$ and $\ln \mathrm{T}_{2}$ on $\ln \left[\mathrm{M}_{\Sigma}\right]$ (Figure 7) was used to estimate sizes of the primary and secondary nuclei. Similar to insulin fibrils, the size of the first nucleus is one monomer, and the size of the secondary nucleus is zero (see Table 2). It should be noted that "one monomer" may correspond to the oligomer structure consisting of 2-6 monomers, if we did not start from the pure monomers in solution.

\section{3. $A \beta 40$}

The experimental kinetics data on the formation of amyloid aggregates, which we used to calculate the sizes of nuclei of amyloid protofibrils formed by A $\beta 40$, were taken from paper [60]. For all the experimental data, we obtained $L_{\text {rel }} \gg 0.2$, which means that the process goes by the exponential mechanism of fibril formation (Figure 8). For $A \beta 40$, we obtained that the size of the primary nucleus is two monomers, and the secondary nucleus size is one monomer.
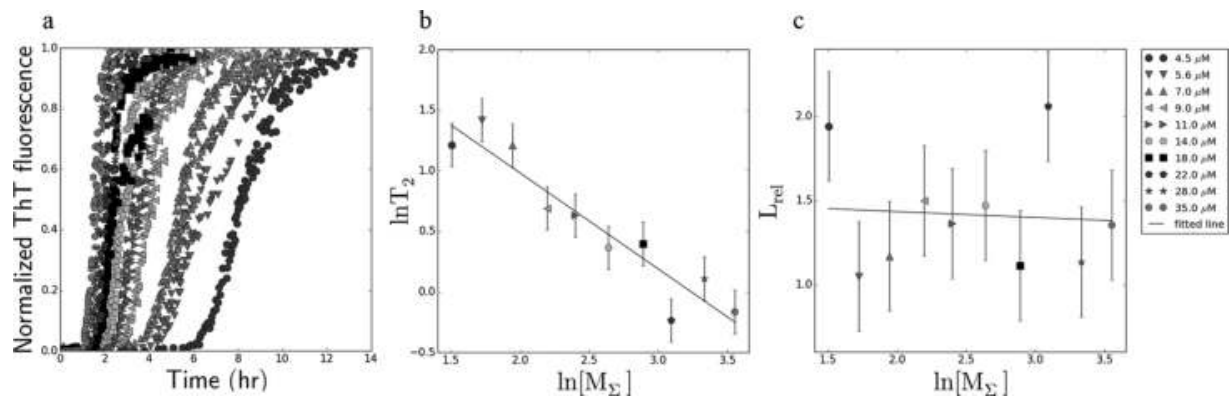

Figure 8. (a) Dependence of normalized ThT fluorescence on time for A $\beta 40$ [60]. The approximation [Insert figure caption here] line calculated for A $\beta 40$ amyloid formation with experimental data from (a) by the least-squares method plotted in coordinates (b): $\left\{\ln \mathrm{T}_{2}, \ln \left[\mathrm{M}_{\Sigma}\right]\right\}$ and (c): $\left\{\mathrm{L}_{\mathrm{rel}}, \ln \left[\mathrm{M}_{\Sigma}\right]\right\} ;\left[\mathrm{M}_{\Sigma}\right]$ is in $\mu \mathrm{M}, \mathrm{T}_{2}$ in hours. The tangent coefficients of the approximation lines are $-0.79 \pm 0.18$ for $\mathrm{d}\left(\ln \mathrm{T}_{2}\right) / \mathrm{d}\left(\ln \left[\mathrm{M}_{\Sigma}\right]\right)$ and $-0.03 \pm 0.32$ for $\mathrm{d}\left(\mathrm{L}_{\mathrm{rel}}\right) / \mathrm{d}\left(\ln \left[\mathrm{M}_{\Sigma}\right]\right)$; the errors are computed with the Student's coefficients corresponding to 0.95 confidence level. 


\section{4. $\mathrm{A} \beta 42$}

The exponential growth with branching takes place for both $A \beta 40$ and $A \beta 42$ peptides (see Figures 8 and 9). Interestingly, according to our analysis, for $A \beta 42$, the size of the primary nucleus of the amyloid protofibril is larger than that of $A \beta 40$, although the mechanism of amyloid fibril formation must be similar. This is consistent with the recent data, which compares the behavior of the two peptides during the aggregation process [60]. The primary nucleus size for $\mathrm{A} \beta 42$ is three monomers, and the secondary nucleus size is two monomers.
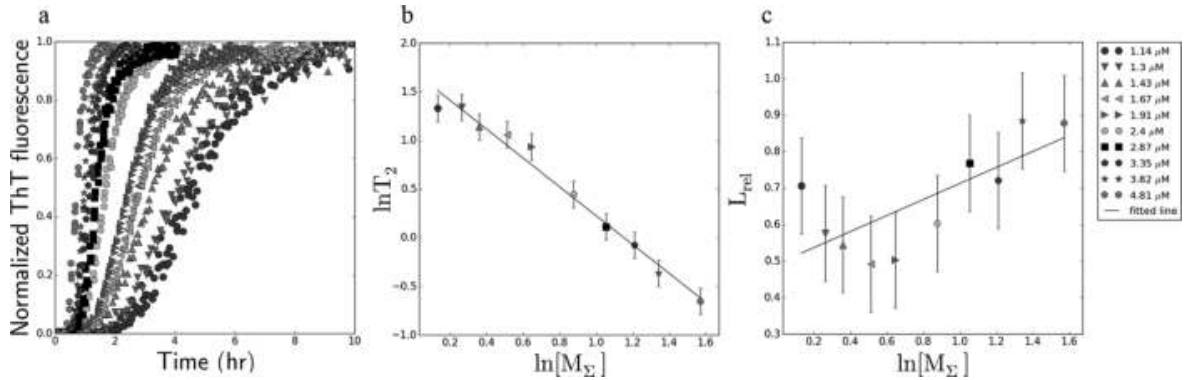

Figure 9. (a) Dependence of normalized ThT fluorescence on time for A $\beta 42$ [61]. The approximation line calculated for A $\beta 42$ amyloid formation with experimental data from (a) by the least-squares method plotted in coordinates (b): $\left\{\ln T_{2}\right.$ $\left.\ln \left[\mathrm{M}_{\Sigma}\right]\right\}$ and (c): $\left\{\mathrm{L}_{\mathrm{rel}}, \ln \left[\mathrm{M}_{\Sigma}\right]\right\} ;\left[\mathrm{M}_{\Sigma}\right]$ is in $\mu \mathrm{M}, \mathrm{T}_{2}$ in hours. The tangent coefficients of the approximation lines are $-1.48 \pm 0.14$ for $\mathrm{d}\left(\ln \mathrm{T}_{2}\right) / \mathrm{d}\left(\ln \left[\mathrm{M}_{\Sigma}\right]\right)$ and $0.22 \pm 0.13$ for $\mathrm{d}\left(\mathrm{L}_{\mathrm{rel}}\right) / \mathrm{d}\left(\ln \left[\mathrm{M}_{\Sigma}\right]\right)$; the errors are computed with the Student's coefficients corresponding to 0.95 confidence level.

\subsection{Human cardiac titin immunoglobulin domain I27 (TI27)}

To determine the sizes of folding nuclei for amyloid formation, calculations were done using the data of aggregation kinetics for the I27 immunoglobulin domain from human cardiac titin (TI27) [62]. The set of data was digitized, and in the case of TI27, part of the curve was excluded from the analysis. It was found that the growth of amyloids formed by TI27 is exponential, that is, in addition to the primary core nucleation secondary nucleation by a branching mechanism also takes place, accelerating the formation of new fibrils.
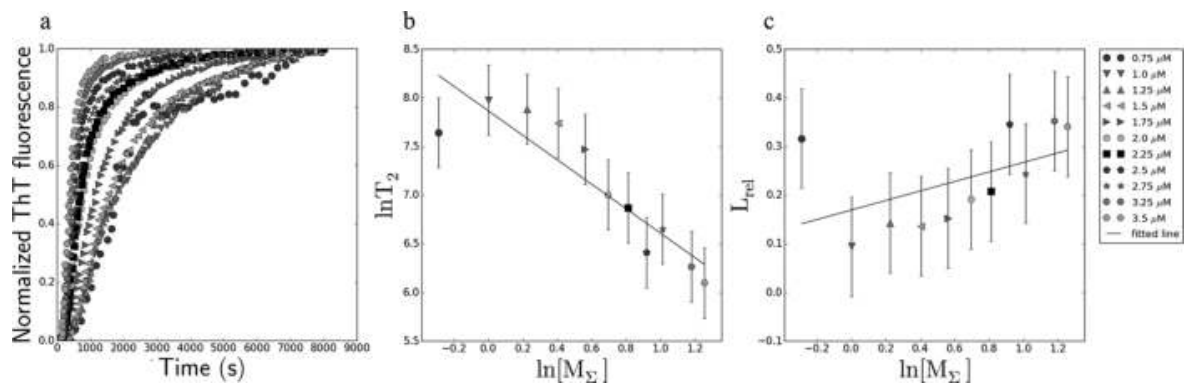
Figure 10. (a) Dependence of normalized ThT fluorescence on time for TI27 [62]. (b) Dependence of $\ln T_{2}$ on $\ln \left[\mathrm{M}_{\Sigma}\right]$ for TI27. This was used to calculate the size of the secondary nucleus. The slope of the fitted line is about -1.26 which, according to formulas from [33], indicates that the size of the secondary nucleus is $\mathrm{n}_{2}=1.52 \pm 0.36$. (c) Dependence of $L_{\text {rel }}$ on $\ln \left[M_{\Sigma}\right]$ for TI27. The slope of the fitted line is -0.1 , that is, $L_{\text {rel }}$ is dependent on $\ln \left[M_{\Sigma}\right]$. Some $L_{\text {rel }}$ values exceed 0.2 and, according to [33], cannot be explained by the linear model. The size of the primary nucleus was calculated based on the size of the secondary nucleus, and the slope of the straight line is $2.42 \pm 0.46$. The errors are computed with the Student's coefficients corresponding to 0.95 confidence level.

It is seen from Figure 10c that though the values of $\mathrm{L}_{\text {rel }}$ for TI27 do not exceed $0.2, \mathrm{~L}_{\text {rel }}$ depends on $\ln \left[\mathrm{M}_{\Sigma}\right]$ and so, as mentioned above, cannot occur by the linear mechanism. This effect can be explained by the presence of second nucleation cores with $n_{2}=1.52 \pm 0.36$ (Figure 10b). It is interesting that in spite of the difference in the aggregation mechanism, the size of the primary nucleation core for TI27 was $2.42 \pm 0.46$ (Figure 10c) similar (within error) to the primary nucleation core, $3.46 \pm 0.28$ (Figure 5), calculated for actin [50].

\section{Concluding remarks}

Amyloid fibril polymorphism suggests various ways of their formation from monomers through different oligomers (intermediates of various sizes and shapes) to mature fibrils of different morphology. It is very likely that the surface of the formed fibrils can serve as a matrix for lateral polymerization of monomers/oligomers on it, as reported in the literature [47, 63, 64]. The above complicates the explanation of the mechanism of formation of fibrils and the proposal of a single scheme of polymerization for many proteins. It should be noted that a combination of several mechanisms is quite possible and can be observed sometimes in experiments.

Thus, despite some common features of formation of fibrils of different proteins, each of them has its own specific route for fibrillogenesis, which is originally determined by the amino acid sequence of the protein and is strongly dependent on environmental conditions and seed addition. It is clear that there is need for a serious analysis of data on various proteins to explain various routes for fibril formation that will help identify conditions that affect the initial formation paths (lag time) and find ways to block the entire process, which is an important task in the prevention and treatment of diseases associated with amyloid deposits.

A detailed study of molecular and cellular processes underlying the development of proteinopathy, search for targets for drug development and the use of the obtained data in the development of methods for early diagnosis are essential for the successful treatment and prevention of common diseases. Due to the new generation of neuroprotective drugs acting directly on the pathogenesis of the disease, all components of the cascade of pathological key protein aggregation proteinopathy can be regarded as potential targets. We believe that the combination of theoretical and experimental approaches employed throughout our research is particularly useful and productive for obtaining new important results. 


\section{Acknowledgements}

We thank T.B. Kuvshinkina, E.I. Grigorashvili, M. Yu. Suvorina, A.V. Finkelstein, and A.K. Surin for assistance in the manuscript preparation. This study was supported by the Russian Science Foundation No. 14-14-00536.

\section{Author details}

Oxana V. Galzitskaya*, Nikita V. Dovidchenko and Olga M. Selivanova

*Address all correspondence to: ogalzit@vega.protres.ru

Institute of Protein Research, Russian Academy of Sciences, Pushchino, Moscow Region, Russia

\section{References}

[1] Chiti F, Webster P, Taddei N, Clark A, Stefani M, Ramponi G, et al. Designing conditions for in vitro formation of amyloid protofilaments and fibrils. Proc. Natl. Acad. Sci. U. S. A. 1999;96:3590-4.

[2] Fändrich M, Fletcher MA, Dobson CM. Amyloid fibrils from muscle myoglobin. Nature. 2001;410:165-6.

[3] Bucciantini M, Calloni G, Chiti F, Formigli L, Nosi D, Dobson CM, et al. Prefibrillar amyloid protein aggregates share common features of cytotoxicity. J. Biol. Chem. 2004;279:31374-82.

[4] Kayed R, Head E, Thompson JL, McIntire TM, Milton SC, Cotman CW, et al. Common structure of soluble amyloid oligomers implies common mechanism of pathogenesis. Science. 2003;300:486-9.

[5] Ivanova MI, Sievers SA, Sawaya MR, Wall JS, Eisenberg D. Molecular basis for insulin fibril assembly. Proc. Natl. Acad. Sci. U. S. A. 2009;106:18990-5.

[6] Dobson CM. Protein misfolding, evolution and disease. Trends Biochem. Sci. 1999;24:329-32.

[7] Rochet JC, Lansbury PT. Amyloid fibrillogenesis: themes and variations. Curr. Opin. Struct. Biol. 2000;10:60-8.

[8] Uversky VN, Gillespie JR, Fink AL. Why are "natively unfolded" proteins unstructured under physiologic conditions? Proteins. 2000;41:415-27. 
[9] Tenidis K, Waldner M, Bernhagen J, Fischle W, Bergmann M, Weber M, et al. Identification of a penta- and hexapeptide of islet amyloid polypeptide (IAPP) with amyloidogenic and cytotoxic properties. J. Mol. Biol. 2000;295:1055-71.

[10] von Bergen M, Friedhoff P, Biernat J, Heberle J, Mandelkow EM, Mandelkow E. Assembly of tau protein into Alzheimer paired helical filaments depends on a local sequence motif ((306)VQIVYK(311)) forming beta structure. Proc. Natl. Acad. Sci. U. S. A. 2000;97:5129-34.

[11] Ivanova MI, Sawaya MR, Gingery M, Attinger A, Eisenberg D. An amyloid-forming segment of beta2-microglobulin suggests a molecular model for the fibril. Proc. Natl. Acad. Sci. U. S. A. 2004;101:10584-9.

[12] Tsolis AC, Papandreou NC, Iconomidou VA, Hamodrakas SJ. A consensus method for the prediction of "aggregation-prone" peptides in globular proteins. Plos One. 2013;8:e54175.

[13] Walsh I, Seno F, Tosatto SCE, Trovato A. PASTA 2.0: an improved server for protein aggregation prediction. Nucleic Acids Res. 2014;42:W301-7.

[14] Fernandez-Escamilla A-M, Rousseau F, Schymkowitz J, Serrano L. Prediction of sequence-dependent and mutational effects on the aggregation of peptides and proteins. Nat. Biotechnol. 2004;22:1302-6.

[15] Emily M, Talvas A, Delamarche C. MetAmyl: a METa-predictor for AMYLoid proteins. Plos One. 2013;8:e79722.

[16] Maurer-Stroh S, Debulpaep M, Kuemmerer N, Lopez de la Paz M, Martins IC, Reumers $\mathrm{J}$, et al. Exploring the sequence determinants of amyloid structure using positionspecific scoring matrices. Nat. Methods. 2010;7:237-42.

[17] Garbuzynskiy SO, Lobanov MY, Galzitskaya OV. FoldAmyloid: a method of prediction of amyloidogenic regions from protein sequence. Bioinform. Oxf. Engl. 2010;26:326-32.

[18] Ahmed AB, Znassi N, Château M-T, Kajava AV. A structure-based approach to predict predisposition to amyloidosis. Alzheimers Dement. J. Alzheimers Assoc. 2015;11:68190.

[19] Dovidchenko NV, Galzitskaya OV. Computational approaches to identification of aggregation sites and the mechanism of amyloid growth. Adv. Exp. Med. Biol. 2015;855:213-39.

[20] Oosawa F, Asakura S, Hotta K, Imai N, Ooi T. G-F transformation of actin as a fibrous condensation. J. Polym. Sci. 1959;37:323-36.

[21] Hofrichter J, Ross PD, Eaton WA. Kinetics and mechanism of deoxyhemoglobin S gelation: a new approach to understanding sickle cell disease. Proc. Natl. Acad. Sci. U. S. A. $1974 ; 71: 4864-8$. 
[22] Ferrone FA, Hofrichter J, Sunshine HR, Eaton WA. Kinetic studies on photolysisinduced gelation of sickle cell hemoglobin suggest a new mechanism. Biophys. J. 1980;32:361-80.

[23] Frieden C, Goddette DW. Polymerization of actin and actin-like systems: evaluation of the time course of polymerization in relation to the mechanism. Biochemistry. 1983;22:5836-43.

[24] Goldstein RF, Stryer L. Cooperative polymerization reactions. Analytical approximations, numerical examples, and experimental strategy. Biophys. J. 1986;50:583-99.

[25] Ruschak AM, Miranker AD. Fiber-dependent amyloid formation as catalysis of an existing reaction pathway. Proc. Natl. Acad. Sci. U. S. A. 2007;104:12341-6.

[26] Griffith JS. Self-replication and scrapie. Nature. 1967;215:1043-4.

[27] Prusiner SB. Novel proteinaceous infectious particles cause scrapie. Science. 1982;216:136-44.

[28] Prusiner SB. Molecular biology of prion diseases. Science. 1991;252:1515-22.

[29] Jarrett JT, Lansbury PT. Seeding “one-dimensional crystallization" of amyloid: a pathogenic mechanism in Alzheimer's disease and scrapie? Cell. 1993;73:1055-8.

[30] Collins SR, Douglass A, Vale RD, Weissman JS. Mechanism of prion propagation: amyloid growth occurs by monomer addition. Plos Biol. 2004;2:e321.

[31] Morris AM, Watzky MA, Agar JN, Finke RG. Fitting neurological protein aggregation kinetic data via a 2-step, minimal/“Ockham's Razor” model: the Finke-Watzky mechanism of nucleation followed by autocatalytic surface growth. Biochemistry. 2008;47:2413-27.

[32] Knowles TPJ, Waudby CA, Devlin GL, Cohen SIA, Aguzzi A, Vendruscolo M, et al. An analytical solution to the kinetics of breakable filament assembly. Science. 2009;326:1533-7.

[33] Dovidchenko NV, Finkelstein AV, Galzitskaya OV. How to determine the size of folding nuclei of protofibrils from the concentration dependence of the rate and lagtime of aggregation. I. Modeling the amyloid protofibril formation. J. Phys. Chem. B. 2014;118:1189-97.

[34] Fändrich M, Schmidt M, Grigorieff N. Recent progress in understanding Alzheimer's $\beta$-amyloid structures. Trends Biochem. Sci. 2011;36:338-45.

[35] Fändrich M, Meinhardt J, Grigorieff N. Structural polymorphism of Alzheimer Abeta and other amyloid fibrils. Prion. 2009;3:89-93.

[36] Petkova AT, Leapman RD, Guo Z, Yau W-M, Mattson MP, Tycko R. Self-propagating, molecular-level polymorphism in Alzheimer's beta-amyloid fibrils. Science. 2005;307:262-5. 
[37] Kajava AV, Baxa U, Steven AC. Beta arcades: recurring motifs in naturally occurring and disease-related amyloid fibrils. FASEB J. 2010;24:1311-9.

[38] Suvorina MY, Selivanova OM, Grigorashvili EI, Nikulin AD, Marchenkov VV, Surin AK, et al. Studies of polymorphism of amyloid- $\beta 42$ peptide from different suppliers. J. Alzheimers Dis. 2015;47:583-93.

[39] Paravastu AK, Qahwash I, Leapman RD, Meredith SC, Tycko R. Seeded growth of betaamyloid fibrils from Alzheimer's brain-derived fibrils produces a distinct fibril structure. Proc. Natl. Acad. Sci. U. S. A. 2009;106:7443-8.

[40] Lashuel HA, Hartley DM, Petre BM, Wall JS, Simon MN, Walz T, et al. Mixtures of wild-type and a pathogenic (E22G) form of Abeta40 in vitro accumulate protofibrils, including amyloid pores. J. Mol. Biol. 2003;332:795-808.

[41] Waugh DF. Regeneration of insulin from insulin fibrils by the action of alkali. J. Am. Chem. Soc. 1948;70:1850-7.

[42] Nielsen L, Khurana R, Coats A, Frokjaer S, Brange J, Vyas S, et al. Effect of environmental factors on the kinetics of insulin fibril formation: elucidation of the molecular mechanism. Biochemistry. 2001;40:6036-46.

[43] Nielsen L, Frokjaer S, Brange J, Uversky VN, Fink AL. Probing the mechanism of insulin fibril formation with insulin mutants. Biochemistry. 2001;40:8397-409.

[44] Nielsen L, Frokjaer S, Carpenter JF, Brange J. Studies of the structure of insulin fibrils by Fourier transform infrared (FTIR) spectroscopy and electron microscopy. J. Pharm. Sci. 2001;90:29-37.

[45] Dzwolak W, Ravindra R, Lendermann J, Winter R. Aggregation of bovine insulin probed by DSC/PPC calorimetry and FTIR spectroscopy. Biochemistry. 2003;42:1134755.

[46] Jansen R, Grudzielanek S, Dzwolak W, Winter R. High pressure promotes circularly shaped insulin amyloid. J. Mol. Biol. 2004;338:203-6.

[47] Jansen R, Dzwolak W, Winter R. Amyloidogenic self-assembly of insulin aggregates probed by high resolution atomic force microscopy. Biophys. J. 2005;88:1344-53.

[48] Selivanova OM, Suvorina MY, Dovidchenko NV, Eliseeva IA, Surin AK, Finkelstein $\mathrm{AV}$, et al. How to determine the size of folding nuclei of protofibrils from the concentration dependence of the rate and lag-time of aggregation. II. Experimental application for insulin and LysPro insulin: aggregation morphology, kinetics, and sizes of nuclei. J. Phys. Chem. B. 2014;118:1198-206.

[49] Binger KJ, Pham CLL, Wilson LM, Bailey MF, Lawrence LJ, Schuck P, et al. Apolipoprotein C-II amyloid fibrils assemble via a reversible pathway that includes fibril breaking and rejoining. J. Mol. Biol. 2008;376:1116-29. 
[50] Sept D, McCammon JA. Thermodynamics and kinetics of actin filament nucleation. Biophys. J. 2001;81:667-74.

[51] Brange J, Dodson GG, Edwards DJ, Holden PH, Whittingham JL. A model of insulin fibrils derived from the $X$-ray crystal structure of a monomeric insulin (despentapeptide insulin). Proteins. 1997;27:507-16.

[52] Pease LF, Sorci M, Guha S, Tsai D-H, Zachariah MR, Tarlov MJ, et al. Probing the nucleus model for oligomer formation during insulin amyloid fibrillogenesis. Biophys. J. 2010;99:3979-85.

[53] Brange J, Andersen L, Laursen ED, Meyn G, Rasmussen E. Toward understanding insulin fibrillation. J. Pharm. Sci. 1997;86:517-25.

[54] Turnell WG, Finch JT. Binding of the dye congo red to the amyloid protein pig insulin reveals a novel homology amongst amyloid-forming peptide sequences. J. Mol. Biol. 1992;227:1205-23.

[55] Nayak A, Sorci M, Krueger S, Belfort G. A universal pathway for amyloid nucleus and precursor formation for insulin. Proteins. 2009;74:556-65.

[56] Ahmad A, Millett IS, Doniach S, Uversky VN, Fink AL. Partially folded intermediates in insulin fibrillation. Biochemistry. 2003;42:11404-16.

[57] Ahmad A, Uversky VN, Hong D, Fink AL. Early events in the fibrillation of monomeric insulin. J. Biol. Chem. 2005;280:42669-75.

[58] Vestergaard B, Groenning M, Roessle M, Kastrup JS, van de Weert M, Flink JM, et al. A helical structural nucleus is the primary elongating unit of insulin amyloid fibrils. Plos Biol. 2007;5:e134.

[59] Podestà A, Tiana G, Milani P, Manno M. Early events in insulin fibrillization studied by time-lapse atomic force microscopy. Biophys. J. 2006;90:589-97.

[60] Meisl G, Yang X, Hellstrand E, Frohm B, Kirkegaard JB, Cohen SIA, et al. Differences in nucleation behavior underlie the contrasting aggregation kinetics of the $A \beta 40$ and A 342 peptides. Proc. Natl. Acad. Sci. U. S. A. 2014;111:9384-9.

[61] Cohen SIA, Linse S, Luheshi LM, Hellstrand E, White DA, Rajah L, et al. Proliferation of amyloid- $\beta 42$ aggregates occurs through a secondary nucleation mechanism. Proc. Natl. Acad. Sci. U. S. A. 2013;110:9758-63.

[62] Wright CF, Teichmann SA, Clarke J, Dobson CM. The importance of sequence diversity in the aggregation and evolution of proteins. Nature. 2005;438:878-81.

[63] Manno M, Mauro M, Craparo EF, Podestà A, Bulone D, Carrotta R, et al. Kinetics of different processes in human insulin amyloid formation. J. Mol. Biol. 2007;366:258-74. 
[64] Loksztejn A, Dzwolak W. Vortex-induced formation of insulin amyloid superstructures probed by time-lapse atomic force microscopy and circular dichroism spectroscopy. J. Mol. Biol. 2010;395:643-55.

[65] Fodera V, Librizzi F, Groenning M, van de Weert M, Leone M. Secondary Nucleation and Accessible Surface in Insulin Amyloid Fibril Formation. J. Phys. Chem. B 2008, 112, 3853-3858. 